\title{
Pleural Fluid Adenosine Deaminase (Pfada) in the Diagnosis of Tuberculous Effusions in a Low Incidence Population
}

\author{
David T. Arnold ${ }^{1}$, Rahul Bhatnagar ${ }^{1,2}$, Lynette D. Fairbanks ${ }^{3}$, Natalie Zahan-Evans ${ }^{2}$, \\ Amelia O. Clive ${ }^{1,2}$, Anna J. Morley ${ }^{2}$, Andrew R. L. Medford ${ }^{2}$, Nicholas A. Maskell ${ }^{1,2}$ \\ 1 Academic Respiratory Unit, School of Clinical Sciences, University of Bristol, Bristol, United Kingdom, \\ 2 North Bristol Lung Centre, Southmead Hospital, Bristol, United Kingdom, 3 Purine Research Laboratory, \\ St Thomas' Hospital, London, United Kingdom \\ * arnold.dta@gmail.com
}

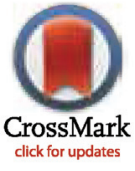

\section{G OPEN ACCESS}

Citation: Arnold DT, Bhatnagar R, Fairbanks LD, Zahan-Evans N, Clive AO, Morley AJ, et al. (2015) Pleural Fluid Adenosine Deaminase (Pfada) in the Diagnosis of Tuberculous Effusions in a Low Incidence Population. PLoS ONE 10(2): e0113047. doi:10.1371/journal.pone. 0113047

Academic Editor: Joan A Caylà, Public Health Agency of Barcelona, SPAIN

Received: August 7, 2014

Accepted: October 18, 2014

Published: February 3, 2015

Copyright: @ 2015 Arnold et al. This is an open access article distributed under the terms of the Creative Commons Attribution License, which permits unrestricted use, distribution, and reproduction in any medium, provided the original author and source are credited.

Data Availability Statement: All relevant data are within the paper and its Supporting Information files.

Funding: The authors received no specific funding for this work.

Competing Interests: The authors have declared that no competing interests exist.

\begin{abstract} Introduction

Previous studies have assessed the diagnostic ability of pleural fluid adenosine deaminase (pfADA) in detecting tuberculous pleural effusions, with good specificity and sensitivity reported. However, in North Western Europe pfADA is not routinely used in the investigation of a patient with an undiagnosed pleural effusion, mainly due to a lack of evidence as to its utility in populations with low mycobacterium tuberculosis (mTB) incidence.
\end{abstract}

\section{Methods}

Patients presenting with an undiagnosed pleural effusion to a tertiary pleural centre in South-West England over a 3 year period, were prospectively recruited to a pleural biomarker study. Pleural fluid from consecutive patients with robust 12-month follow up data and confirmed diagnosis were sent for pfADA analysis.

\section{Results}

Of 338 patients enrolled, 7 had confirmed tuberculous pleural effusion (2\%). All mTB effusions were lymphocyte predominant with a median pfADA of $72.0 \mathrm{IU} / \mathrm{L}$ (range- 26.7 to 91.5 ) compared to a population median of $12.0 \mathrm{IU} / \mathrm{L}$ (range- 0.3 to 568.4 ). The optimal pfADA cut off was $35 \mathrm{IU} / \mathrm{L}$, which had a negative predictive value (NPV) of $99.7 \%(95 \% \mathrm{Cl}$; $98.2-$ $99.9 \%)$ for the exclusion of mTB, and sensitivity of $85.7 \%(95 \% \mathrm{Cl} ; 42.2-97.6 \%)$ with an area under the curve of $0.88(95 \% \mathrm{Cl} ; 0.732-1.000)$.

\section{Discussion}

This is the first study examining the diagnostic utility of pfADA in a low mTB incidence area. The chance of an effusion with a pfADA under $35 \mathrm{IU} / \mathrm{L}$ being of tuberculous aetiology was negligible. A pfADA of over $35 \mathrm{IU} / \mathrm{L}$ in lymphocyte-predominant pleural fluid gives a strong suspicion of mTB. 


\section{Introduction}

Pleural fluid adenosine deaminase (pfADA) measurement is commonly used in countries with a moderate to high incidence of mycobacterium tuberculosis (mTB). In these areas it is often used routinely in the investigation of undiagnosed pleural effusions, or to supplement standard pleural fluid analysis where a tuberculous effusion is suspected. ADA is a purine catabolic enzyme that catalyses the conversion of adenosine to inosine and is particularly abundant in lymphoid tissue[1]. In comparison to the other investigations for mTB, pfADA can be quickly analysed and is relatively non-invasive to obtain with thoracocentesis. Direct visualisation of acid fast bacilli in pleural fluid has low yield rates of between $5-10 \%[2,3]$ and culture results from pleural fluid and sputum only have a sensitivity of 50\% and 30\% respectively, as well as taking up to 8 weeks to perform $[4,5]$. In countries where the BCG was used nationally the Mantoux test has limited utility. The gold standard for diagnosis of pleural mTB is thoracoscopic pleural biopsy, with pick-up rates approaching $100 \%[6]$, but in some patients this invasive test may be inappropriate.

In the UK, and other North-Western European countries, pfADA has limited use outside of specialist centres, in part due to a lack of evidence where mTB incidence is low[7]. We report a prospective study examining the utility of pfADA in an area with an mTB incidence of 7.8 per $100,000[8]$.

\section{Methods}

\section{Patients}

Consecutive patients referred to a tertiary pleural centre with an undiagnosed pleural effusion had blood, pleural fluid, and clinical and demographic information collected prospectively. The study received ethical approval from the South West regional ethics committee (REC number $08 / \mathrm{H} 0102 / 11$ ) and all patients provided written informed consent. In addition to routine baseline pleural investigations pleural fluid was centrifuged, and the supernatant stored at $-70^{\circ} \mathrm{c}$. All patients were assigned a final diagnosis at either death or 12 months (whichever came sooner). The final diagnosis was agreed by two independent respiratory consultant physicians based on all the available clinical information. Any areas of contention were re-examined to reach consensus. Notably, pfADA results were not available at the time of diagnosis.

\section{Diagnostic criteria}

Pre-defined diagnostic criteria were used to reach a 12 month diagnosis. Tuberculous pleural effusion was diagnosed in the presence of any of the following: positive fluid or tissue culture for mycobacterium tuberculosis (mTB); acid fast bacilli confirmed in sputum, pleural fluid or pleural tissues; or in patients with a strong clinical and radiological suspicion of TB pleuritis who had a positive quantiferon and showed resolution of the presenting pleural effusion after 6 months anti-TB therapy. See S1 Appendix for full details and other diagnostic criteria.

\section{Pleural fluid analysis}

Routine pleural fluid analysis was completed at the time of study enrolment and included protein, glucose, LDH, pH, microscopy, culture, cytology and flow cytometry with cytogenetics. Light's criteria were used to distinguish exudative from transudative effusions[9]. Predominant pleural cell types were defined based on British Thoracic Society guidelines[10]. Specifically a lymphocyte-predominant effusion was defined as the presence of over $50 \%$ lymphocytes in the absence of any malignant cells or $\geq 10 \%$ eosinophils, in which case the effusion was deemed malignant or eosinophilic respectively. 
pfADA was measured 'en bloc' post patient recruitment after a median of 41 months (range 25-63 months) of storage at $-70^{\circ} \mathrm{C}$, by a single investigator blinded to patient diagnosis. A photodiode array ultraviolet detector was used with a non-Giusti method. Before analysis (to reduce the risk of infection) all samples were thawed then filtered using individual Sterifix 0.2um luer lock filters in a category 3 laboratory and were refrozen before transit. See S2 Appendix for full details on pfADA analysis.

\section{Statistical analysis}

Statistical analysis was performed using SPSS 21.0 statistical software (Chicago, IL, USA). pfADA was non-parametric so was described using medians and ranges. Demographic and biochemical factors affecting pfADA were assessed using multiple linear regression analysis with backwards selection. Variables with a p value $>0.1$ were removed from the model. The accuracy of pfADA as a diagnostic test was assessed using standard sensitivity, specificity, positive predictive values (PPV) and negative predictive values (NPV) with 95\% confidence intervals.

\section{Results}

\section{Patient demographics and aetiology of pleural effusions}

338 consecutive patients with stored pleural fluid from undiagnosed pleural effusions were entered into a pleural biomarkers study between December 2008 and February 2012 and had their pleural fluid sent for ADA analysis. The cohort had a mean age of 70.6 (range 18-96) and was $65 \%$ male ( 218 male vs 120 female).

Table 1 shows the breakdown of all effusions, with malignant (51\%) and parapneumonic (21\%) aetiologies forming the majority. 79\% (267/338) of all cause effusions were exudates as defined by Light's criteria. Of 71 parapneumonic effusions, 12 were diagnosed as bacterial empyema and 39 were diagnosed as complicated parapneumonic effusions (CPPE). Reasons for CPPE diagnosis included low pleural $\mathrm{pH}$ or loculations on USS $(\mathrm{n}=28)$, positive gram stain or culture of pleural fluid $(n=3)$, culture or histological findings of pleural biopsy $(n=4)$, and CT evidence of pleural infection with radiological evidence of resolution following treatment $(n=4)$.

Table 2 shows the details of the 7 patients diagnosed with a tuberculous effusion (2\%) including basis for diagnosis and baseline demographic, biochemical and sensitivity data. Five had a culture positive diagnosis of $\mathrm{mTB}, 1$ had granulomatous tissue seen at biopsy and a positive quantiferon, and 1 had a clinical and radiological suspicion of $\mathrm{mTB}$ with a positive quantiferon. All had resolution of their pleural effusion after 6 months of anti-TB therapy. No patients were found to have atypical mycobacteria or antibiotic resistance on culture. There were no cases of concurrent HIV infection, although two patients were receiving immunosuppressive therapy for autoimmune disease. Of the 7 patients with mTB all had lymphocyte predominant exudative pleural effusions.

\section{pfADA in all effusion types}

The median pfADA level was $12.0 \mathrm{IU} / \mathrm{L}$ (range 1.3 to 568.4). Fig. 1 shows the distribution of pfADA by aetiology for all effusions. Median levels of pfADA were highest in empyema (104.9 IU/L, range 17.2-568.4) followed by mTB (median 72.0 IU/L, range 26.7 to 91.5). Multiple linear regression was used to assess the effect of demographic, pleural and serum factors on pfADA levels. After backward elimination, patient age $(R=-0.161, P=0.049)$, pleural protein $(\mathrm{R}=0.249, \mathrm{P}=0.002)$ and pleural $\mathrm{LDH}(\mathrm{R}=0.009, \mathrm{P}<0.001)$ were independently linked to pfADA level. Notably there was no correlation between the storage time of pleural fluid and pfADA. 
Table 1. Aetiology of pleural effusion across the cohort (n-338).

\begin{tabular}{ll}
\hline Causes & N (\%) \\
\hline Malignancy & $172(51 \%)$ \\
Lung & 56 \\
MPM & 42 \\
Breast & 24 \\
Ovarian & 14 \\
Gastrointestinal & 7 \\
Lymphoma & 8 \\
Head and neck & 5 \\
Other & 16 \\
Transudates & $44(13 \%)$ \\
CCF & 33 \\
Hepatic & 7 \\
Renal & 4 \\
Parapneumonic & $71(21 \%)$ \\
Simple & 20 \\
Complicated & 39 \\
Empyema & 12 \\
Mycobacterium Tuberculosis & $7(2 \%)$ \\
Other exudates & $39(12 \%)$ \\
BAPE & 15 \\
Inflammatory pleuritis & 11 \\
Trauma & 4 \\
Pulmonary embolus & 3 \\
Other & 3 \\
Idiopathic & 6 \\
\hline & $5(1 \%)$ \\
\hline
\end{tabular}

BAPE- Benign Asbestos Related Pleural Effusion, CCF- Congestive Cardiac Failure, MPM- Malignant Pleural Mesothelioma.

doi:10.1371/journal.pone.0113047.t001

Based on previous literature[11] and confirmatory results from our receiver operating curve (ROC) a cut off of $35 \mathrm{IU} / \mathrm{L}$ (greater than or equal to) was chosen to differentiate 'low' from 'high' pfADA. Using this cut off there were 36 patients who fell into the high pfADA group, all of whom had exudative effusions (see Table 3). Of these, 7 were lymphocyte predominant with 6 tuberculous effusions and 1 CPPE (see Fig. 2). Two cases of non small cell lung cancer (NSCLC) and 4 cases of malignant pleural mesothelioma (MPM) had pfADA $\geq 35 \mathrm{IU} / \mathrm{L}$ compared to group medians of $10.6 \mathrm{IU} / \mathrm{L}$ and $16.8 \mathrm{IU} / \mathrm{L}$ respectively. The only causes of a pfADA over 100 were 7 cases of empyema which were all strongly neutrophil-predominant ( $\geq 80 \%$ minimum). None of the 44 transudative effusions (defined by Light's criteria) had a pfADA $\geq 35 \mathrm{IU} / \mathrm{L}$ (median $5.7 \mathrm{IU} / \mathrm{L}$ ). Notably, all 8 cases of lymphoma had pfADA $<35 \mathrm{IU} / \mathrm{L}$ (median 20.4 IU/L, range 8.5-29.7). There was one case of $\mathrm{mTB}$ which fell into the low pfADA group (26.7 IU/L), a 41 year old male who was a current smoker.

\section{pfADA as a diagnostic test}

Using a cut off of $35.0 \mathrm{IU} / \mathrm{L}$, for all effusion types $(\mathrm{n}=338)$, the sensitivity of pfADA for detecting mTB was $85.7 \%$ with a specificity of $90.9 \%$ and NPV of $99.7 \%$. Area under the curve was 0.883 (95\% CI; 0.732-1.000). Full results are shown in Table 4. 
Table 2. Details of patients diagnosed with tuberculous pleural effusion.

\begin{tabular}{|c|c|c|c|c|c|c|}
\hline $\begin{array}{l}\text { Agel } \\
\text { Sex }\end{array}$ & $\begin{array}{l}\text { Smoking } \\
\text { status }\end{array}$ & $\begin{array}{l}\text { pfADA } \\
\text { (IU/L) }\end{array}$ & $\begin{array}{l}\text { Predominant cell ( } \% \\
\text { on differential) }\end{array}$ & Diagnostic evidence & $\begin{array}{l}\text { Sensitivities to anti- } \\
\text { TB antibiotics }\end{array}$ & $\begin{array}{l}\text { Resolution with } \\
\text { anti-TB therapy? }\end{array}$ \\
\hline $37 \mathrm{M}$ & Never & 89.0 & Lymphocytic (85\%) & $\begin{array}{l}\text { Pleural biopsy, sputum and pleural fluid } \\
\text { grew mTB }\end{array}$ & Fully & Yes \\
\hline $70 \mathrm{~F}$ & Never & 37.9 & Lymphocytic (60\%) & $\begin{array}{l}\text { Pleural biopsy, sputum and pleural fluid } \\
\text { grew mTB }\end{array}$ & Fully & Yes \\
\hline $45 \mathrm{M}$ & Never & 72.0 & Lymphocytic (100\%) & $\begin{array}{l}\text { Biopsy demonstrated granulomatous } \\
\text { tissue. No growth on culture. Positive } \\
\text { quantiferon }\end{array}$ & $\mathrm{n} / \mathrm{a}$ & Yes \\
\hline $40 \mathrm{M}$ & Never & 73.3 & Lymphocytic (70\%) & Pleural biopsy grew mTB & Fully & Yes \\
\hline $81 \mathrm{M}$ & Ex & 91.5 & Lymphocytic (95\%) & Pleural fluid grew mTB & Fully & Yes \\
\hline $41 \mathrm{M}$ & Current & 26.7 & Lymphocytic (75\%) & BAL grew mTB & Fully & Yes \\
\hline $38 \mathrm{M}$ & Never & 35.0 & Lymphocytic (80\%) & $\begin{array}{l}\text { Clinical and radiological suspicion of mTB } \\
\text { but no growth on culture. Positive } \\
\text { quantiferon. }\end{array}$ & $\mathrm{n} / \mathrm{a}$ & Yes \\
\hline
\end{tabular}

BAL- bronchoalveolar lavage, mTB- mycobacterium tuberculosis.

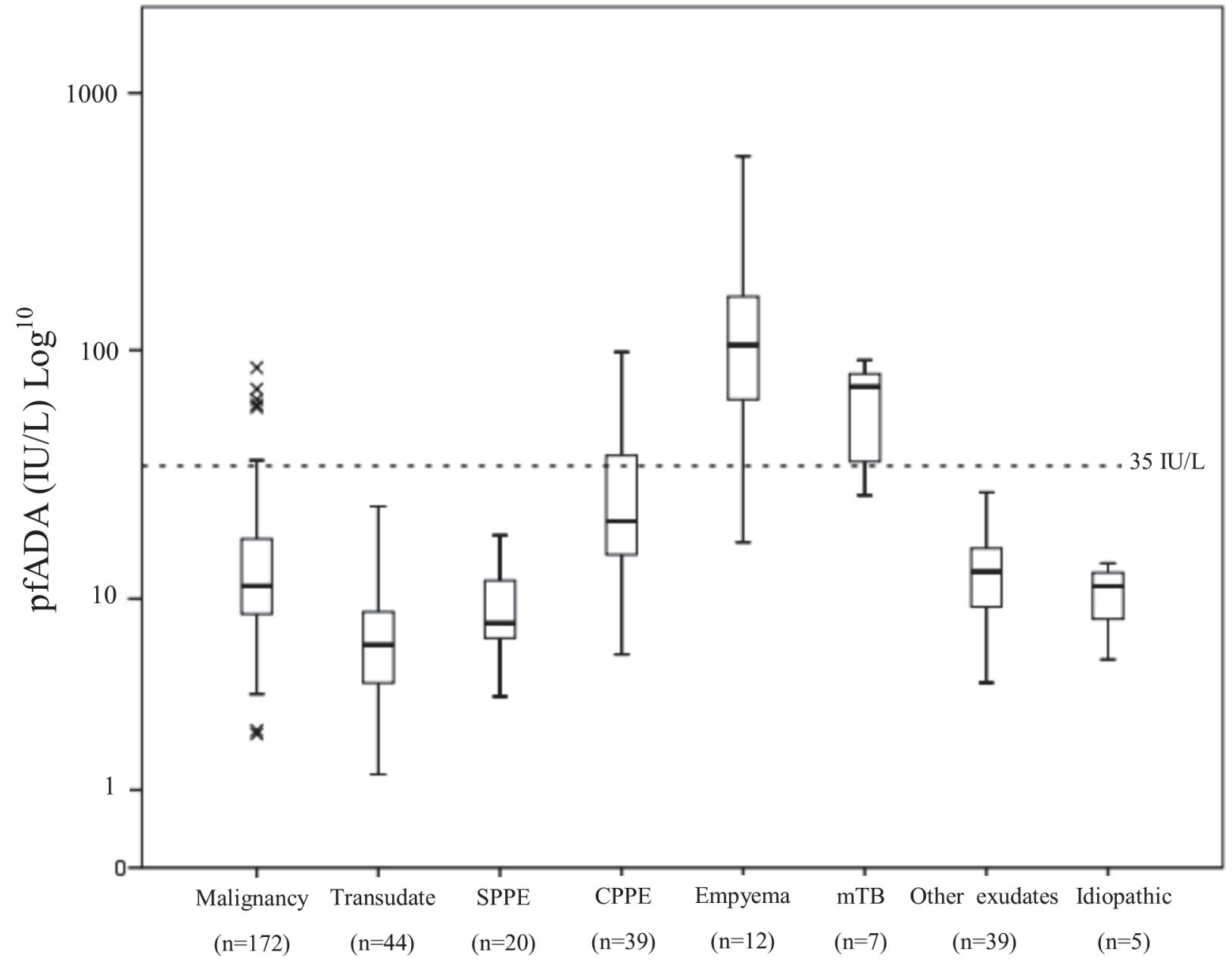

Fig 1. Boxplot of pfADA levels by diagnostic category for all effusions $(n=338)$. Abbreviations; SPPE-Simple parapneumonic effusion, CPPEComplicated parapneumonic effusion, mTB-mycobacterium tuberculosis. 
Table 3. Causes of a high pfADA ( $\geq 35 \mathrm{IU} / \mathrm{L})$.

\begin{tabular}{llllll}
\hline Aetiology & N- & pfADA range (IU/L) & Lymphocyte predominant & Neutrophil predominant & Mean pleural pH (Range) \\
\hline Mycobacterium tuberculosis & 6 & $35.0-91.5$ & $6(100 \%)$ & $0(0 \%)$ & $7.35(7.10-7.40)$ \\
Complex parapneumonic effusion & 13 & $35.7-98.1$ & $1(8 \%)$ & $9(69 \%)$ & $6.96(6.68-7.34)$ \\
Empyema & 11 & $54.7-568.4$ & $0(0 \%)$ & $10(91 \%)$ & $6.91(6.67-7.24)$ \\
Malignant pleural mesothelioma & 4 & $59.7-70.4$ & $0(0 \%)$ & $1(25 \%)$ & $7.19(7.17-7.33)$ \\
Non small cell lung cancer & 2 & $36.8-85.4$ & $0(0 \%)$ & $1(50 \%)$ & $7.20(7.09-7.31)$ \\
\hline
\end{tabular}

doi:10.1371/journal.pone.0113047.t003

Table 4. Cross tabulation for the diagnostic performance of pfADA in all effusions $(n=338)$.

\begin{tabular}{|c|c|c|c|}
\hline & Mycobacterium tuberculosis & No mycobacterium tuberculosis & \\
\hline \multirow[t]{3}{*}{$\mathrm{pfADA} \geq 35 \mathrm{IU} / \mathrm{L}$} & 6 & 30 & Positive predictive value \\
\hline & & & $16.7 \%$ \\
\hline & & & (95\% Cl; 6.4-32.8\%) \\
\hline \multirow[t]{6}{*}{ pfADA $<35$ IU/L } & 1 & 301 & Negative predictive value \\
\hline & & & $99.7 \%$ \\
\hline & & & (95\% Cl; 98.2-99.9\%) \\
\hline & Sensitivity & Specificity & \\
\hline & $85.7 \%$ & $90.9 \%$ & \\
\hline & (95\% Cl; 42.2-97.6\%) & (95\% Cl; 87.3-98.8\%) & \\
\hline
\end{tabular}

doi:10.1371/journal.pone.0113047.t004

When using the same cut-off in lymphocytic effusions alone ( $\mathrm{n}=98)$ NPV falls to $98.9 \%$. Sensitivity remains the same and specificity improves to $98.9 \%$ with a greater area under the curve, 0.923 (95\% CI; 0.770-1.000). Full results are shown in Table 5. See S3 Appendix for receiver operating curves (ROC) of both all effusion and lymphocytic effusions alone.

\section{Discussion}

The aim of this study was to test the ability of pfADA to aid in the diagnosis of tuberculous pleural effusions in an area where mTB incidence is low. In 2013 Public Health England (formerly the Health Protection Agency) reported the rate of mTB in South-West of England, where this study is based, as 7.8 per 100,000, in comparison to a national UK average of 14.6 per 100,000 [8]. This is a similar incidence to other North-Western European counties e.g. France (8.2), Denmark (7.4), Germany (5.6)[12]. The main study finding was that a pfADA of less than $35 \mathrm{IU} / \mathrm{L}$, regardless of effusion type, made a diagnosis of mTB very unlikely with a NPV of 99.7\%. Additionally, patients with a lymphocytic effusion and a pfADA over $35 \mathrm{IU} / \mathrm{L}$ had a high likelihood of mTB ( $85 \%$ compared to a cohort prevalence of $2 \%$ ).

\section{Previous literature}

There have been several meta-analyses assessing the utility pfADA measurement, the largest carried out by Liang and colleagues who assessed 63 studies of varying size (28 to 600 patients). Despite including a large number of patients $(n=8093)$, their high values for sensitivity and specificity have little relevance to a low incidence population such as in the UK-the prevalence of $\mathrm{mTB}$ in our cohort was $2 \%$ (7/338) compared to an average prevalence of $34.5 \%$ in the metaanalysis. Differing methods of pfADA analysis (Giusti's colorimetric method vs non-Giusti) are likely to have influenced the conclusions further still. 


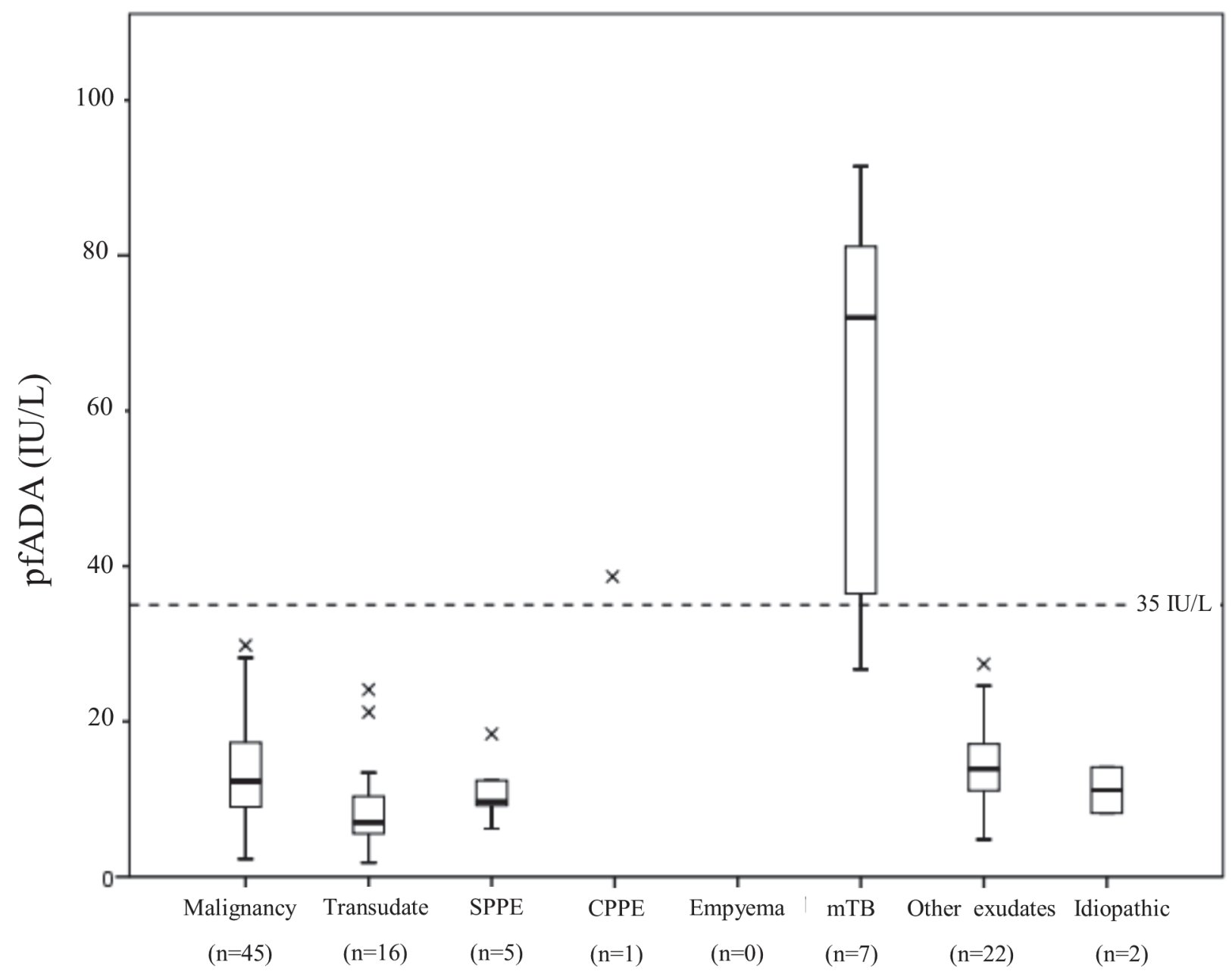

Fig 2. Boxplot of pfADA levels by diagnostic category for lymphocyte predominant effusions $(\mathbf{n}=\mathbf{9 8})$. Abbreviations; SPPE-Simple parapneumonic effusion, CPPE-Complicated parapneumonic effusion, mTB—mycobacterium tuberculosis.

doi:10.1371/journal.pone.0113047.g002

Porcel et al attempted to address the issue of varying methodologies by recruiting a large number of patients to a single trial in an intermediate incidence area (30 per 100,000)[11]. The authors used a Bayes' formula technique to model a low prevalence situation. They concluded that as prevalence fell the positive predictive value would also fall, but negative predictive values would remain high. However, although this technique can allow for changes in mTB prevalence it makes assumptions as to the type of effusions that would then predominate. Another Spanish based study[13] used three time periods where incidence rates were different (thanks to a very effective $\mathrm{mTB}$ control programme) to examine the effect on pfADA's diagnostic ability. They also concluded that the negative predictive value remains high, especially when used in combination with lymphocyte-predominance data. It should be noted that the lowest mTB rates in this study were still four times that of our cohort.

\section{Interpreting a low pfADA}

In a low incidence population the likelihood of $\mathrm{mTB}$ is small, but patients with undiagnosed pleural effusions after routine investigations[14] and unable to undergo pleural biopsy may be given empirical anti-TB therapy if clinical suspicion remains high. This approach risks severe 
Table 5. Cross tabulation for the diagnostic performance of pfADA in lymphocytic effusions $(n=98)$.

\begin{tabular}{|c|c|c|c|}
\hline & Mycobacterium tuberculosis & No mycobacterium tuberculosis & \\
\hline \multirow[t]{3}{*}{ pfADA $\geq 35 \mathrm{IU} / \mathrm{L}$} & 6 & 1 & Positive predictive value \\
\hline & & & $85.7 \%$ \\
\hline & & & (95\% Cl; 42.2-97.6\%) \\
\hline \multirow[t]{6}{*}{$\operatorname{pfADA}<35 \mathrm{IU} / \mathrm{L}$} & 1 & 90 & Negative predictive value \\
\hline & & & $98.9 \%$ \\
\hline & & & (95\% Cl; 94.0-99.8\%) \\
\hline & Sensitivity & Specificity & \\
\hline & $85.7 \%$ & 98.9 & \\
\hline & (95\% Cl; 42.2-97.6\%) & (95\% Cl; 94.0-99.8\%) & \\
\hline
\end{tabular}

doi:10.1371/journal.pone.0113047.t005

drug side effects in patients where the most likely alternative diagnosis is malignancy. In our study the likelihood of mTB fell to $0.3 \%$ in the context of a pfADA less than $35 \mathrm{IU} / \mathrm{L}$ in all cause effusions, and $1.0 \%$ in lymphocytic effusions. The strong performance of pfADA as a 'rule out' test means the use of empirical therapy is unlikely to be of benefit in this population group. As well as reducing patient harm, avoiding presumptive therapy is likely to be cost effective.

Previous literature would suggest low pfADA values should be viewed with caution in certain scenarios if clinical suspicion remains high. These include active smokers and increasing age[15]. Previous studies have attempted to stratify pfADA levels to patient age in tuberculous effusions $[16,17]$. Unfortunately this study does not have the power to identify age specific reference ranges but also found that pfADA fell with age after multivariate analysis. Unlike some other diagnostic tests for mTB immunosuppression does not appear to affect pfADA levels[18].

\section{Interpreting a high pfADA}

We also demonstrated that a pfADA over $35 \mathrm{IU} / \mathrm{L}$ detects mTB with good sensitivity and specificity. A patient with an effusion of malignant aetiology had only a $3 \%(6 / 172)$ chance of having a high pfADA and $0 \%(0 / 172)$ chance of the effusion also being lymphocyte predominant. The ability of pfADA to act as a 'rule in' test for mTB was greatly improved by consideration of the predominant cell type, increasing the specificity from $91 \%$ in all cause effusions to $99 \%$ in lymphocytic effusions.Six cases (2 NSCLC and 4 MPM) of malignant cause effusions had a high pfADA, although five appeared to have a co-existent pleural infection In other studies, high pfADA values have been reported in lymphoma[11, 19, 20], although all lymphoma cases in our cohort had pfADA values of less than 35 IU/L. Differentiating between mTB and lymphoma can be challenging, and additional tests such as lymphocyte subset analysis may help to distinguish one from the other[21]. A third of parapneumonic effusions had pfADA values of over $35 \mathrm{IU} / \mathrm{L}$ and in the case of empyema the result could be over 15 times the upper limit of normal. The phenomena of exceptionally high pfADA (e.g. $>250 \mathrm{u} / \mathrm{L}$ ) in empyema has been well documented[11, 13, 15].

\section{The place of pfADA in clinical practice in a low incidence population}

Despite the good performance of pfADA in this study we would only support its use in certain clinical scenarios. Firstly, in patients for whom the cause of a pleural effusion is unclear after initial investigations but more invasive pleural biopsy is inappropriate the focus would be to use pfADA to exclude mTB and thereby avoid the need for empirical treatment. 
Secondly, in patients with lymphocytic effusions where mTB is strongly suspected but traditional investigations have proved inconclusive a high pfADA infers a strong likelihood of $\mathrm{mTB}$ and could prompt empirical anti-TB treatment. A high pfADA might raise the possibility of $\mathrm{mTB}$ where it previously seemed unlikely. However, a patient with a pfADA over 35 IU/L should still undergo a biopsy where possible to obtain microbiological confirmation and sensitivity data. This is especially important in areas of increasing resistance to first line antibiotics.

\section{Study limitations}

Despite the large number of patients included in our study, due to low prevalence there were only 7 cases of mTB. Although the low numerator affects estimates of sensitivity, it has much less impact on the specificity and negative predictive value, and so assessments of the test's ability to rule out mTB are likely to be accurate. Additionally, we only encountered cases of lymphocyte-predominant tuberculous effusion so are unable to comment on tuberculous effusions where neutrophils predominate. pfADA analysis was performed at an external laboratory, by an investigator blinded to patient diagnosis, and performed 'en bloc' postrecruitment. For this reason samples were stored frozen for a significant period of time and filtered before transit. Although pfADA has been shown to be highly stable[22] some studies have shown a small decline in the yield of pfADA after extended frozen storage[23]. However, storage time was not correlated with pfADA in our cohort. This study analysed the total pfADA from patient samples i.e. the sum of its isoforms $\mathrm{ADA}_{1}$ and $\mathrm{ADA}_{2}$. Some studies have supported the analysis of $\mathrm{ADA}_{2}$ alone as a marginally more specific analyte, as it reduces the false positive effect from neutrophilic effusions[24, 25]. However, the improved specificity of this isoform is superseded by consideration of the predominant cell type which we recommend for all baseline pleural fluid analysis. Not all clinical laboratories routinely report the differential cell counts of pleural fluid which affects the generalisability of this recommendation. We hope this study gives further weight to the value of cytology reports that routinely include differential cell counts.

\section{Summary}

This is the first prospective study of the diagnostic accuracy of pfADA in a population of low $\mathrm{mTB}$ incidence. We have shown that a pfADA under $35 \mathrm{IU} / \mathrm{L}$ makes a diagnosis of mTB highly unlikely. A pfADA of over $35 \mathrm{IU} / \mathrm{L}$ in lymphocyte predominant effusions makes mTB the most likely diagnosis but does not replace pleural biopsy as the gold standard investigation. Based on these data we propose clinical scenarios where pfADA could be used in the work up of undiagnosed pleural effusions in areas of low mTB incidence. Further prospective studies to validate our findings would be beneficial.

\section{Supporting Information}

S1 Appendix. Diagnostic protocol for undiagnosed pleural effusions. (DOCX)

S2 Appendix. Adenosine deaminase assay. (DOCX)

S3 Appendix. Receiver operating curves. (DOCX) 


\section{Acknowledgments}

We would like to thank all members of the pleural biomarkers study who collected data used in this report, and Elena Domenech and Andrew Lovering for their help in the preparation and filtering of the pleural fluid.

\section{Author Contributions}

Conceived and designed the experiments: DA NM. Performed the experiments: LF. Analyzed the data: DA AC. Contributed reagents/materials/analysis tools: LF AJM NZE. Wrote the paper: DA RB NM. Diagnosis of patients: ARLM NM.

\section{References}

1. Villegas MV, Labrada LA, Saravia NG (2000) Evaluation of polymerase chain reaction, adenosine deaminase, and interferon-gamma in pleural fluid for the differential diagnosis of pleural tuberculosis. Chest; 118(5):1355-64. PMID: 11083686

2. Gopi A, Madhavan SM, Sharma SK, Sahn SA (2007) Diagnosis and treatment of tuberculous pleural effusion in 2006. Chest; 131(3):880-9. PMID: 17356108

3. Valdes L, Alvarez D, San Jose E, Penela P, Valle JM, et al (1998). Tuberculous pleurisy: a study of 254 patients. Arch Intern Med; 158(18):2017-21. PMID: 9778201

4. Seibert AF, Haynes J Jr, Middleton R, Bass JB Jr (1991) Tuberculous pleural effusion. Twenty-year experience. Chest; 99(4):883-6. PMID: 1901261

5. Chakrabarti B, Ryland I, Sheard J, Warburton CJ, Earis JE (2006) The role of Abrams percutaneous pleural biopsy in the investigation of exudative pleural effusions. Chest; 129(6):1549-55. PMID: 16778273

6. Diacon AH, Van de Wal BW, Wyser C, Smedema JP, Bezuidenhout J, et al (2003) Diagnostic tools in tuberculous pleurisy: a direct comparative study. Eur Respir J; 22(4):589-91. PMID: 14582908

7. McGrath EE, Anderson PB (2010) Diagnostic tests for tuberculous pleural effusion. Eur J Clin Microbiol Infect Dis; 29(10):1187-93. doi: 10.1007/s10096-010-0986-z PMID: 20556468

8. Public Health England; Tuberculosis in the UK- 2013 report. Available: http://www.hpa.org.uk/webc/ HPAwebFile/HPAweb_C/1317139689583. Accessed July 2014.

9. Light RW, Macgregor MI, Luchsinger PC, Ball WC Jr (1972) Pleural effusions: the diagnostic separation of transudates and exudates. Ann Intern Med; 77(4):507-13. PMID: 4642731

10. Maskell NA, Butland RJ, Pleural Diseases Group SoCCBTS (2003) BTS guidelines for the investigation of a unilateral pleural effusion in adults. Thorax; 58 Suppl 2:ii8-17. PMID: 12728146

11. Porcel JM, Esquerda A, Bielsa S (2010) Diagnostic performance of adenosine deaminase activity in pleural fluid: a single-center experience with over 2100 consecutive patients. Eur J Intern Med; 21 (5):419-23. doi: 10.1016/j.ejim.2010.03.011 PMID: 20816597

12. World Health Organization (WHO) estimates of tuberculosis incidence by country (2012) (sorted by country). Available: http://wwwhpaorguk/webc/HPAwebFile/HPAweb_C/1317140584754. Accessed July 2014

13. Garcia-Zamalloa A, Taboada-Gomez J (2012) Diagnostic accuracy of adenosine deaminase and lymphocyte proportion in pleural fluid for tuberculous pleurisy in different prevalence scenarios. PLoS One; 7(6):e38729. doi: 10.1371/journal.pone.0038729 PMID: 22723878

14. Hooper C, Lee YC, Maskell N, Group BTSPG (2010) Investigation of a unilateral pleural effusion in adults: British Thoracic Society Pleural Disease Guideline. Thorax; 65 Suppl 2:ii4-17. PMID: 20696692

15. Lee SJ, Kim HS, Lee SH, Lee TW, Lee HR, et al (2014) Factors Influencing Pleural Adenosine Deaminase Level in Patients With Tuberculous Pleurisy. Am J Med Sci. doi: 10.1097/MAJ.0b013e31828ffcd6 PMID: 25545390

16. Kapisyzi P, Argjiri D, Aliko A, Beli J, Vakeflliu Y, et al (2011) The Use of Different Cutoff Values of ADA Liquid Level in Diagnosis of Tuberculous Pleurisy in Countries With Different Incidence of Tuberculosis (Abstract) Chest; 140(4_MeetingAbstracts):703A. doi: 10.1378/chest.1108296

17. Tay TR, Tee A (2013) Factors affecting pleural fluid adenosine deaminase level and the implication on the diagnosis of tuberculous pleural effusion: a retrospective cohort study. BMC Infect Dis; 13:546. doi: 10.1186/1471-2334-13-546 PMID: 24238276 
18. Chung JH, Kim YS, Kim SI, Park K, Park MS, et al (2004) The diagnostic value of the adenosine deaminase activity in the pleural fluid of renal transplant patients with tuberculous pleural effusion. Yonsei Med J; 45(4):661-4. PMID: 15344207

19. Yao CW, Wu BR, Huang KY, Chen HJ (2014) Adenosine deaminase activity in pleural effusions of lymphoma patients. QJM. PMID: 25524909

20. Antonangelo L, Vargas FS, Genofre EH, Oliveira CM, Teixeira LR, et al (2012) Differentiating between tuberculosis-related and lymphoma-related lymphocytic pleural effusions by measuring clinical and laboratory variables: is it possible? J Bras Pneumol; 38(2):181-7. PMID: 22576425

21. Bhatnagar R, Clive A, Zahan-Evans N, Morley A, Virgo P, et al (2013) The clinical utility of pleural lymphocyte subset analysis in undiagnosed effusions (abstract). Thorax; 68(3):170-1.

22. Antonangelo L, Vargas FS, Almeida LP, Acencio MM, Gomes FD, et al (2006) Influence of storage time and temperature on pleural fluid adenosine deaminase determination. Respirology; 11(4):488-92. PMID: 16771922

23. Bielsa S, Esquerda A, Palma RM, Criado A, Porcel JM (2014) Influence of storage time on pleural fluid adenosine deaminase activity. Clin Lab; 60(3):501-4. PMID: 24697129

24. Valdes L, San Jose E, Alvarez D, Valle JM (1996) Adenosine deaminase (ADA) isoenzyme analysis in pleural effusions: diagnostic role, and relevance to the origin of increased ADA in tuberculous pleurisy. Eur Respir J; 9(4):747-51. PMID: 8726940

25. Zemlin AE, Burgess LJ, Carstens ME (2009) The diagnostic utility of adenosine deaminase isoenzymes in tuberculous pleural effusions. Int J Tuberc Lung Dis; 13(2):214-20. PMID: 19146750 